Pesq. Vet. Bras. 30(7):533-540, julho 2010

\title{
Intoxicação experimental por monofluoroacetato de sódio em bovinos: aspectos clínicos e patológicos ${ }^{1}$
}

\author{
Vivian A. Nogueira ${ }^{2 \star}$, Ticiana N. França ${ }^{2}$, Tiago C. Peixoto ${ }^{3}$, Saulo A. Caldas ${ }^{4}$, \\ Anibal G. Armién ${ }^{5}$ e Paulo V. Peixoto ${ }^{6}$
}

\begin{abstract}
Nogueira V.A., França T.N., Peixoto T. C., Caldas S.A., Armién A.G. \& Peixoto P.V. 2010. [Experimental poisoning by sodium monofluoroacetate in cattle: Clinical and pathological aspects.] Intoxicação experimental por monofluoroacetato de sódio em bovinos: aspectos clínicos e patológicos. Pesquisa Veterinária Brasileira 30(7):533-540. Projeto Sanidade Animal Embrapa/UFRRJ, Seropédica, RJ 23890-000, Brazil. E-mail: vivianmedvet@yahoo.com.br
\end{abstract}

Sodium monofluoroacetate (MF) was identified, by cromatography, in three of the 12 plants that cause sudden death in cattle in Brazil, including Palicourea marcgravii, the most important plant of this group. A special kind of hydropic-vacuolar degeneration (HVD) of the distal convoluted uriniferous tubules was considered typical for the poisoning by some authors. The objective of this study was to verify if the ingestion of MF causes similar clinical signs and lesion in cattle poisoned by plants that cause sudden death. Six cows received orally 0.5 and $1.0 \mathrm{mg} / \mathrm{kg}$ of $\mathrm{M}$ diluted in $50 \mathrm{~mL}$ of distilled water. Clinically the animals presented tachycardia, engorgement and positive pulse of the jugular vein, abdominal breathing, swaying gait, subtle unbalance, and recumbency with head placed the on the flank. In the agonal phase all the animals in lateral recumbence presented muscular fasciculation, tonic contraction, peddling movements, opisthotonus, nystagmus, and died between 2 and 14 minutes. At postmortem examination, the heart auricles, jugular and pulmonary veins were moderately distended and engorged with blood. Slight to moderate edema of the subserosa was seen around the gall bladder and the duodenum near to the pancreas. At light microscopy, HVD associated with nuclear picnosis of the epithelial cells of the distal convoluted uriniferous tubules was present in all animals. Ultrastructurally, the cell lesion represents formation of cytosolic vacuoles, likely due to accumulation of water. Coagulation necrosis of individual or groups of hepatocytes and slight hepatic congestion secondary to the venous stasis were also observed. Hydropic-vacuolar degeneration has been observed in cases of poisoning by many substances, which cause acute tubular nephrosis, however not restricted to the distal renal tubules and without nuclear picnosis. This study demonstrates that the HVD in the kidney can be caused by MF and, in analogy, the compound should be considered responsible for the death of cattle that ingest toxic plants which cause sudden death in Brazil. Our results can be supportive to studies that will focus on the degradation of MF by rumen bacteria, what might have economic implications, as at least 500.000 cattle die annually by sudden death causing toxic plants in Brazil.

INDEX TERMS: Sodium monofluoroacetate, hydropic-vacuolar degeneration, poisonous plants, cattle, plant poisoning, pathology.

\footnotetext{
${ }^{1}$ Recebido em 19 de novembro de 2009.

Aceito para publicação em 14 de janeiro de 2010.

Parte da Tese de Doutorado do primeiro autor no Curso de Ciências Veterinárias, Universidade Federal Rural do Rio de Janeiro (UFRRJ), Seropédica, RJ.

${ }^{2}$ Departamento de Epidemiologia e Saúde Pública, Instituto de Veterinária, UFRRJ, Seropédica, RJ 23890-000, Brasil. Autor para correspondência: vivianmedvet@yahoo.com.br
}

\footnotetext{
${ }^{3}$ Mestrando do Curso de Pós-Graduação em Medicina Veterinária, UFRRJ, Seropédica, RJ.

${ }^{4}$ Doutorando do Curso de Pós-Graduação em Ciências Veterinárias, UFRRJ, Seropédica, RJ.

5 Department of Veterinary Population Medicine and Minnesota Veterinary Diagnostic Laboratory, CVM, University of Minnesota, USA. E220 VDL, 1333 Gortner Avenue, St Paul, MN 55108.

${ }^{6}$ Departamento de Nutrição Animal e Pastagem, Instituto de Zootecnia, UFRRJ, Seropédica, RJ.
} 
RESUMO.- Monofluoracetato de sódio (MF) foi identificado, por cromatografia, em três das doze plantas que causam morte súbita em bovinos no Brasil, incluindo Palicourea marcgravii, a mais importante desse grupo. Uma lesão considerada típica por alguns autores para intoxicação foi ${ }^{1}$ a degeneração hidrópico-vacuolardos túbulos uriníferos contorcidos distais (DHV). O objetivo deste trabalho foi verificar se a ingestão de MF induz sinais clínicos e lesões similares às observadas nos bovinos intoxicados pelas plantas que causam morte súbita. Seis vacas receberam, por via oral, 0,5 e 1,0mg/kg de MF diluídos em $50 \mathrm{~mL}$ de água destilada. Clinicamente, os animais apresentaram taquicardia, jugular repleta com pulso venoso positivo, respiração abdominal, ligeira perda de equilíbrio, por vezes cambaleavam, deitavam e apoiavam a cabeça no flanco. Na fase final (agônica), todos os animais caíam em decúbito lateral, esticavam os membros, faziam movimentos de pedalagem, apresentavam opistótono, nistagmo, mugidos e a morte ocorria em 2-14 minutos. À necropsia verificaram-se aurículas, jugulares, ázigos e pulmonares moderadamente ingurgitadas. Observaram-se ainda leve a moderado edema da subserosa nos locais de fixação da vesícula biliar no fígado, além de leve edema entre o duodeno e o pâncreas. O exame histopatológico revelou, em todos os animais, desde leve a acentuada DHV das células epiteliais dos túbulos uriníferos contorcidos distais associada à cariopicnose. Com relação à ultraestrutura, observou-se vacuolização do citoplasma devido ao acúmulo de água. Vacuolização e necrose de coagulação individual ou de grupos de hepatócitos e leve congestão hepática secundários à estase venosa também foram observados. DHV tem sido observada em casos de envenenamento por outras substâncias, porém nestes não está restrita aos túbulos distais e não se observa cariopicnose. Este estudo demonstra que a DHV dos túbulos renais de bovinos pode ser causada pelo envenenamento por MF e, por analogia, essa substância deve ser considerada como um dos fatores importantes, senão o mais significativo, implicado na morte dos animais que ingerem plantas que causam morte súbita no Brasil. Isso indica que estudos, que envolvam metabolização de MF por bactérias ruminais, teriam grande aplicabilidade econômica, uma vez que pelo menos 500.000 bovinos morrem anualmente intoxicados por plantas do grupo das que causam morte súbita no Brasil.

TERMOS DE INDEXAÇÃO: Monofluoroacetato de sódio, degeneração hidrópico-vacuolar, plantas tóxicas, bovinos, intoxicação por planta, patologia.

\section{INTRODUÇÃO}

O monofluoroacetato de sódio (MF), ácido monofluoroacético ou 1080, é uma substância tóxica que teve sua fabricação, comercialização e seu uso proibido nos Estados Unidos e Brasil em 1972 e 1982, respectivamente (Balcomb et al. 1983, Adesp 2007). Em outros países como Austrália e Nova Zelândia, seu uso está restrito ao controle de predadores como cães selvagens, raposas, porcos selva- gens e coelhos europeus (Mcllroy 1981, 1982, 1984, Twigg \& King 1991).

O modo de ação do MF baseia-se na formação do fluorocitrato, seu metabólito ativo, formado no organismo por meio da denominada "síntese letal". O fluoroacetato se liga à acetil coenzima A (CoA-SH) para formar fluoroacetil CoA, que substitui o acetil CoA no ciclo de Krebs e reage com citrato sintase para produzir fluorocitrato. Esse composto bloqueia competitivamente a aconitase e impede a conversão do citrato em isocitrato, o que resulta no acúmulo de citrato em vários tecidos. Além disso, há queda na produção de ATP e processos metabólicos dependentes de energia são bloqueados (Peters 1952, Clarke 1991). A morte sobrevém pelo efeito mais intenso sobre o coração em bovinos (Maxie \& Robinson 2007), ovinos (Schultz et al. 1982), eqüinos, caprinos, coelhos e macacos (Chenoweth \& Gilman 1946), ou sobre o SNC em humanos (Gajdusek \& Luther1950), cães, cobaias, ratos e hamsters (Chenoweth \& Gilman 1946). Em algumas espécies como gato e porco doméstico, o efeito se faz sobre ambos (Chenoweth \& Gilman 1946).

O MF é considerado o princípio tóxico de Dichapetalum cymosum, uma importante planta tóxica da África do Sul, conhecida popularmente como Gifblaar, responsável por grande mortalidade de animais de produção (Marais 1944, Chenoweth 1949, Vickery \& Vickery 1973). Mais tarde, esse composto também foi identificado em outras plantas tóxicas que causam morte súbita (Oxylobium spp., Acacia georginae e Gastrolobium spp.) na Austrália (Oerlichs \& McEwan 1962, McEwan 1964). Com base na opinião de Tokarnia \& Döbereiner (1982), temos empregado o termo morte súbita para definir mortes com evolução de poucos minutos, usualmente sem sinais clínicos prévios evidentes.

No Brasil, as numerosas plantas capazes de induzir morte súbita em bovinos são responsáveis, por pelo menos, metade das mortes causadas por plantas tóxicas nessa espécie no país. Ao exame histopatológico, todas as plantas desse grupo determinam o aparecimento de uma lesão muito típica, denominada degeneração hidrópico-vacuolar das células epiteliais dos túbulos uriníferos contorcidos distais (DHV) associada à cariopicnose (Tokarnia et al. 2000). Em três plantas desse grupo (Palicourea marcgravii, Arrabidaea bilabiata e provavelmente Mascagnia rigida) demonstrouse, pelo menos cromatograficamente, a presença de MF (Oliveira 1963, Krebs et al. 1994, Cunha et al. 2006). Contudo, não foi demonstrado se o ácido é ou não responsável pelas mortes dos animais, nem se o mesmo é capaz de induzir as referidas lesões renais. Alguns autores acreditam que outras substâncias que não o MF podem ser importantes na gênese e no desfecho desses casos de morte súbita (Habermehl 1986, Górniak 1988, Kemmerling 1996, González et al. 2000, Coelho et al. 2007).

Dessa forma, pretende-se verificar se a ingestão de MF induz sinais clínicos e lesões similares às observadas em bovinos intoxicados pelas plantas que causam morte súbita. Adicionalmente, a eventual comprovação de que o MF é a substância responsável, ou, pelo menos, o principal fator determinante da morte dos animais que ingeriram 
essas plantas abriria boas possibilidades de implantaremse medidas profiláticas efetivas, uma vez que, na Austrália, verificou-se que bactérias ruminais podem ser modificadas geneticamente para desdobrar o MF e torná-lo inócuo (Gregg et al. 1998).

\section{MATERIAL E MÉTODOS}

Os experimentos foram realizados em março de 2008 nas instalações do Setor de Anatomia Patológica, do Projeto Sanidade Animal, Convênio Embrapa/UFRRJ. Foram utilizadas seis vacas adultas, com pesos entre 376 e $468 \mathrm{~kg}$, mestiças da raça Holandesa, soropositivas para brucelose, sem alterações clínicas e que deveriam ser eutanasiadas para atender à legislação brasileira. Os animais foram mantidos em baias individuais de alvenaria, com piso de cimento, medindo 4,0m x 5,0m, com acesso à água e capim picado (Panicum maximum) à vontade.

Administraram-se, por via oral, doses únicas de $0,5 \mathrm{mg} / \mathrm{kg}$ e $1,0 \mathrm{mg} / \mathrm{kg}$ de monofluoroacetato de sódio (Sigma Aldrich Co) ${ }^{7}$ diluído em $50 \mathrm{~mL}$ de água destilada. Quatro animais foram submetidos à movimentação. O delineamento dos experimentos realizados encontra-se detalhado no Quadro 1.

Quadro 1. Intoxicação experimental por MFa em bovinos. Delineamento experimental

\begin{tabular}{ccccc}
\hline $\begin{array}{c}\text { Bovino } \\
\text { (Reg.SAPb }\end{array}$ & $\begin{array}{c}\text { Peso } \\
(\mathrm{kg})\end{array}$ & $\begin{array}{c}\text { Dose única } \\
(\mathrm{mg} / \mathrm{kg})\end{array}$ & $\begin{array}{c}\text { Quantidade } \\
\text { total de MF }(\mathrm{mg})\end{array}$ & Movimentado \\
\hline $\begin{array}{c}1190 \\
(31213)\end{array}$ & 432 & 0,5 & 216 & Não \\
$\begin{array}{c}1215 \\
(31209) \\
1262\end{array}$ & 468 & 0,5 & 234 & Sim \\
$\begin{array}{c}(31211) \\
1307\end{array}$ & 439 & 0,5 & 220 & Sim \\
$\begin{array}{c}(31212) \\
1327\end{array}$ & 387 & 1,0 & 387 & Sim \\
$(31214)$ & 410 & 1,0 & 410 & Não \\
1358 & 376 & 1,0 & 376 & Sim \\
$(31210)$ & & & &
\end{tabular}

a Monofluoracetato de sódio; ${ }^{b}$ Registrado no Setor de Anatomia Patológica.

Após a morte, os animais foram imediatamente necropsiados. Fragmentos de diversos órgãos foram coletados, fixados em formol $10 \%$, processados rotineiramente para histopatologia e corados pela Hematoxilina e Eosina. Fragmentos de rim foram fixados em glutaraldeído $4 \%$, pós-fixados em tetróxido de ósmio, embebidos em resina (LR-white $₫)^{8}$, seccionados a 70-90nm e observados em microscópio eletrônico de transmissão.

\section{RESULTADOS}

\section{Dose administrada, início dos sintomas e evolução clí- nica}

As seis vacas que receberam 0,5 e $1,0 \mathrm{mg} / \mathrm{kg}$ de MF morreram. Os animais que receberam $0,5 \mathrm{mg} / \mathrm{kg}$ apresen-

\footnotetext{
${ }^{7}$ Sigma-Aldrich, 3050 Spruce Street, St Louis, MO 63103 EUA, http:/ /www.sigma-aldrich.com

8 LR-white $\AA$, Electron Microscopy Science, P.O. Box 550, 1560 Industry Road, Hattfield, PA 19440 England. http://www.emsdiasum.com/ microscopy/technical/support.aspx.
}

taram os primeiros sintomas leves entre 2 horas 5 minutos e 13 horas e 20 minutos. Sinais graves foram observados entre 5 horas e 3 minutos e 19 horas e 55 minutos após a administração do MF. Nos bovinos que receberam 1,0mg/ $\mathrm{kg}$, os primeiros sintomas leves foram observados entre 2 horas e 12 minutos e 3 horas e os sintomas graves da intoxicação entre 2 horas e 7 horas e 10 minutos após a administração do MF. A duração dos sintomas graves ("fase dramática") variou de 2 a 14 minutos.

\section{Quadro clínico geral}

Em todas as vacas observaram-se taquicardia, jugular repleta (Fig.1) com pulso venoso positivo, respiração abdominal e ligeira perda de equilíbrio; por vezes, os animais cambaleavam, apoiavam a cabeça no flanco, levantavam e deitavam em decúbito esternal, repetidamente. Dois animais apresentaram polaquiúria, três tiveram tremores musculares e dois, sialorréia e veias da face ingurgitadas. Observou-se ainda acentuada miose no Bovino 1358. Na "fase dramática", todos os animais deitavam-se e levantavam-se em menor intervalo de tempo, depois caíam em decúbito lateral, esticavam os membros, faziam movimentos de pedalagem, apresentavam respiração ofegante, opistótono, nistagmo, mugidos e morriam rapidamente.

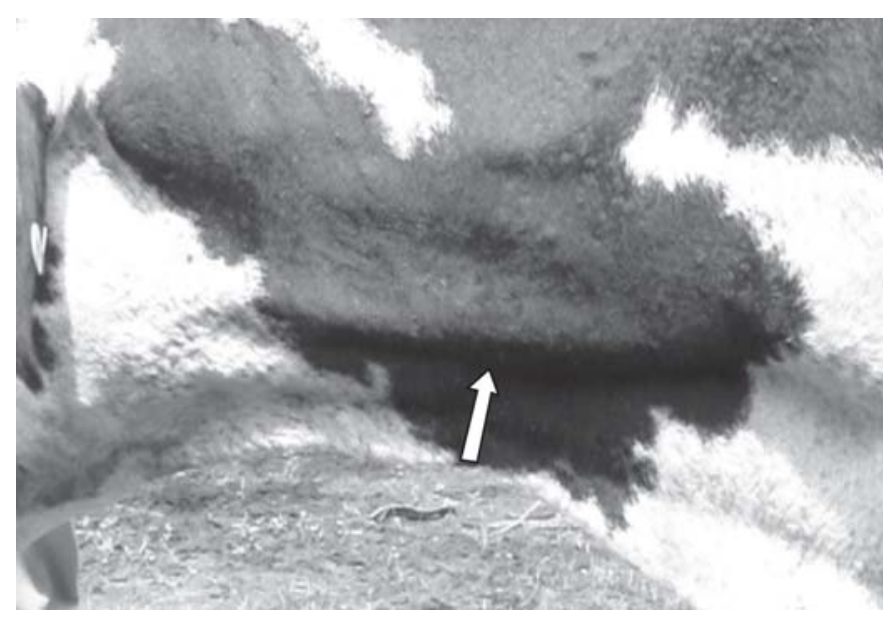

Fig.1. Jugular ingurgitada no Bovino 1262 uma hora antes da morte, na intoxicação experimental por monofluoroacetato de sódio, com dose de $0,5 \mathrm{mg} / \mathrm{kg}$.

\section{Achados de necropsia}

Observaram-se aurículas, veias cava cranial, jugulares, ázigos, costo cervical, subclávia direita e pulmonares leve a moderadamente ingurgitadas (Fig.2). Com exceção do Bovino 1327, os demais apresentaram leve a moderado edema entre a serosa da vesícula biliar e a cápsula hepática, além de leve edema entre o duodeno e o pâncreas. Os demais achados consistiram de raras petéquias no pulmão, leve a acentuado edema pulmonar, leve avermeIhamento da mucosa do abomaso, raras petéquias na mucosa da vesícula biliar, acentuação do padrão lobular do fígado, leve esplenomegalia, leve congestão hepática e leve hidropericárdio. 


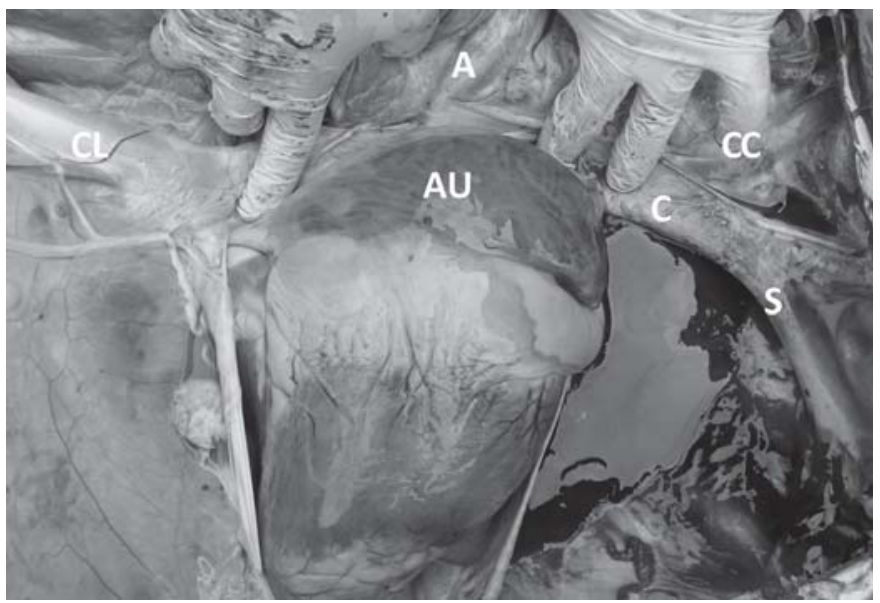

Fig.2. Aurícula $(A U)$ e veias cava caudal $(C L)$ e cranial $(C)$, ázigos direita (A), costo cervival (CC) e subclávia direita (S) ingurgitadas, na intoxicação experimental por monofluoroacetato de sódio, com dose de 1,0mg/kg (Bovino 1358).

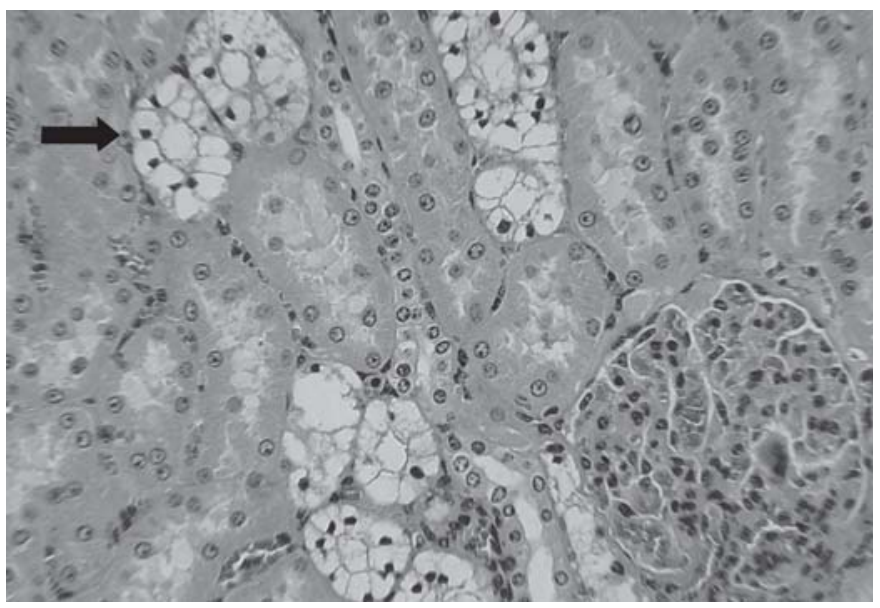

Fig.3. Degeneração hidrópico-vacuolar das células epiteliais dos túbulos uriníferos contorcidos associada à cariopicnose (seta), na intoxicação experimental por monofluoroacetato de sódio, com dose de 0,5mg/kg (Bovino 1215). HE, obj.25x.

\section{Achados histopatológicos}

O exame histopatológico evidenciou, no rim de todos os animais, leve a acentuada degeneração hidrópico-vacuolar dos túbulos uriníferos contorcidos distais e, por vezes, túbulos retos, associada à cariopicnose (Fig.3 e 4). Havia ainda, leve a moderada congestão e leve a moderado infiltrado inflamatório linfo-plasmocitário intersticial. No fígado observaram-se edema do espaço de Disse, leve a moderada congestão, necrose de coagulação paracentral ou necrose individual aleatória, vacuolização de hepatócitos na região centrolobular, leve tumefação difusa dos hepatócitos, leve a moderado infiltrado inflamatório mononuclaear periportal, leve proliferação das vias biliares, leucocitoestase, esférulas eosinofílicas nos sinusóides hepáticos (corpúsculos de choque) e dilatação dos sinusóides principalmente em região centrolobular. No coração observaram-se focos de leve infiltrado inflamatório linfo-plasmocitário e leve congestão, achados não relacionados à intoxicação por MF.

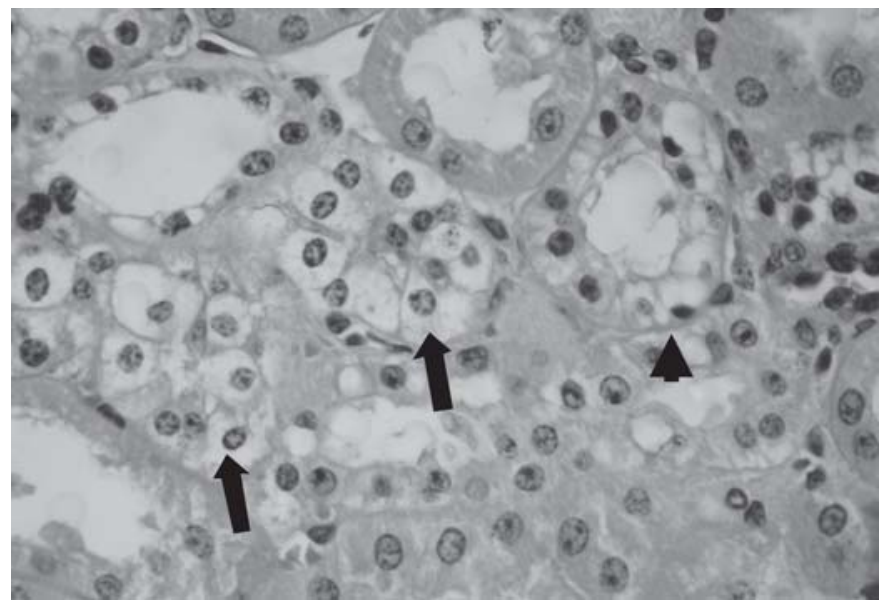

Fig.4. Degeneração hidrópico-vacuolar das células epiteliais dos túbulos uriníferos contorcidos (cabeça de seta: lesão incipiente; seta: lesão mais avançada caracterizada por acentuada cariopicnose), na intoxicação experimental por monofluoroacetato de sódio, na dose de 0,5mg/kg (Bovino 1190). HE, obj.40x.

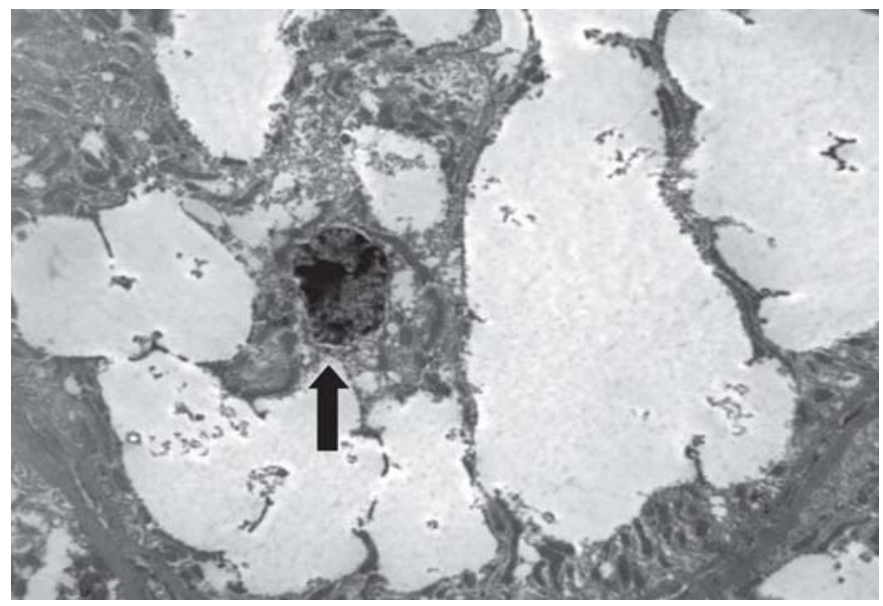

Fig.5. Ultraestrutura de uma célula de túbulo contornado renal mostrando acentuada vacuolização do citoplasma e cariopicnose (seta), na intoxicação experimental por monofluoroacetato de sódio, com dose de $1 \mathrm{mg} / \mathrm{kg}$ (Bovino 1307). 7500x.

Havia ainda leve a moderado edema pulmonar. Nos demais órgãos não foram encontradas alterações significativas.

\section{Achados ultramicroscópicos}

O citoplasma das células epiteliais tubulares afetadas apresentava-se distendido por vacúolos não-lipídicos de 4 $\mu \mathrm{m}$ de diâmetro, não-limitados por membrana, que confluiam e, com freqüência, formavam vacúolos maiores de até $10 \mu \mathrm{m}$. O núcleo dessas células era pequeno e com cromatina muito condensada (cariopicnose) (Fig.5).

\section{DISCUSSÃO}

Todos os bovinos que receberam MF por via oral apresentaram quadro clínico-patológico semelhante, em vários as- 
pectos, ao observado em bovinos que ingerem plantas que causam morte súbita no Brasil.

Os animais apresentaram relutância em andar, queda, dificuldade para levantar, decúbito lateral, membros esticados, tremores musculares, respiração ofegante, movimentos de pedalagem, mugidos, opistótono, nistagmo e morte rápida. Quadro similar foi descrito em experimentos realizados com Palicourea marcgravii (Tokarnia \& Döbereiner 1986) e Pseudocalymma elegans em bovinos (Mello \& Fernandes 1940, Tokarnia et al. 1969, 1993, 1995, Consorte et al. 1994, Helayel 2008), assim como em outros estudos com demais plantas que causam morte súbita (Pacheco \& Carneiro 1932, Döbereiner \& Tokarnia 1959, 1982, 1983, Camargo 1962, Tokarnia \& Döbereiner 1982, Tokarnia et al. 1983, 1985, 1990, 1991, 2000, 2004, Costa et al. 1984, Döbereiner et al. 1984, 1986, Peixoto et al. 1987, Barbosa et al. 2003).

Embora os bovinos que receberam $0,5 \mathrm{mg} / \mathrm{kg}$ de MF tenham começado a apresentar os sintomas antes de serem exercitados, após os exercícios, sempre tiveram os sintomas intensificados, de forma semelhante ao que foi descrito em bovinos intoxicados com plantas que causam morte súbita (Tokarnia et al. 1969, 1981, 1990, 1993, 2000, 2004, Couceiro et al. 1976, Tokarnia \& Döbereiner 1986, Consorte et al. 1994, Gava et al. 1998, Oliveira et al. 2004, Helayel et al. 2009). Apesar do Bovino 1327 (1,0mg/kg) não ter sido exercitado, ele morreu antes do Bovino 1358 que também recebeu $1,0 \mathrm{mg} / \mathrm{kg}$ de MF e foi submetido ao exercício. Tal fato pode ter ocorrido não só em função da susceptibilidade individual, mas também não se pode descartar uma reação ao manejo durante o exame clínico, que faz com que haja uma maior liberação de adrenalina, da qual pode resultar taquicardia, fibrilação e morte. Adicionalmente observaram-se jugulares e grandes vasos ingurgitados, edema pulmonar e dilatação da aurícula do coração direito, alterações já descritas em bovinos intoxicados com P. elegans (Helayel et al. 2009) e por outras plantas que causam morte súbita (Pacheco \& Carneiro 1932, Döbereiner \& Tokarnia 1959, 1982, 1983, Camargo 1962, Tokarnia \& Döbereiner 1982, Tokarnia et al. 1983, 1985, 2000, 2004, Costa et al. 1984, Döbereiner et al. 1984, 1986, Peixoto et al. 1987, Barbosa et al. 2003). Estas alterações são observadas em animais que morrem de insuficiência cardíaca aguda (Jones et al. 1983).

A intoxicação por MF nos bovinos do presente estudo determinou o aparecimento da clássica degeneração hidrópico-vacuolar nos túbulos uriníferos contorcidos distais associada à cariopicnose. Antes da reprodução das lesões renais agora demonstradas, já havia evidências circunstanciais de que o MF seria a causa, ou pelo menos um dos compostos importantes na determinação da morte dos animais que ingerem plantas que causam morte súbita. Essa constatação comprova, pela primeira vez, que o MF induz a DHV dos túbulos uriniferos contorcidos distais, o que indica que estudos, que envolvam metabolização de MF por bactérias ruminais, teriam grande aplicabilidade econômica, uma vez que pelo menos 500.000 bovinos morrem anualmente intoxicados por plantas do grupo das que causam morte súbita no Brasil (Tokarnia 2009).

Não obstante, algumas considerações podem ser feitas a respeito da DHV. Embora tumefação celular e degeneração hidrópica sejam comuns no epitélio tubular, à exceção das descrições sobre lesões renais associadas à ingestão de plantas que causam morte súbita no Brasil (Tokarnia et al. 2000), não encontramos referências a esse tipo especial de lesão.

De fato, degeneração hidrópica vacuolar tem sido observada em casos de envenenamento por diversas substâncias como dioxano (Jones \& Hunt 1983) e selenito de sódio (Khattab 2007), porém, neste caso, a alteração não está restrita aos túbulos distais e não cursa com evidente cariopicnose. Nos trabalhos experimentais ou em envenenamentos acidentais ou intencionais também não encontramos menção específica a esse tipo de lesão. Outras substâncias, como solução de ácido tartárico (Friedman \& Kaplan 1943), cisplatina (Fillastre \& Raguenez-Viotte 1989), glicerol 50\% (Rodrigo et al. 2004) e dicromato de potássio (Cristofori et al. 2007), causam DHV dos túbulos uriníferos contorcidos proximais, sem comprometer os túbulos distais. Outros compostos isolados dessas plantas, como tanóides (Guimarães 1934), saponinas (Guimarães 1934, Mello \& Fernandes 1940, Barnes \& Gillbert 1960), alcalóides (Guimarães 1934, Barnes \& Gilbert 1960, Gagnin \& Maravalhas 1969, Morita et al. 1989), ácidos málico, palicúrico e mioctônico (Peckolt 1934), ácidos salicílico e D-metoxibenzóico (Cascon \& Mors 1962), alcalóides N-metiltyramina (Cascon \& Mors 1962, Kemmerling 1996), cafeína (Górniak 1988), 2-metiltetrahidro-â-carbolina (Kemmerling 1996), salicilato de metila, cristais de oxalato de cálcio (Coelho et al. 2007) e outros, não induzem aos quadros clínicos acima mencionados nem ao aparecimento de DHV.

$\mathrm{O}$ fato de humanos e animais intoxicados por MF desenvolverem variáveis graus de azotemia (e uremia) até o ponto de ocorrer insuficiência renal, é indicação segura de que o ácido realmente lesa o rim, provavelmente durante 0 processo de excreção (Chung 1984). Pode-se inferir que em humanos esse tipo de lesão não ocorra ou esteja associada a alterações ainda mais graves (necrose coagulativa) ou mais "chamativas" no que diz respeito à morfologia, exatamente como se observa em eqüinos intoxicados por $P$. marcgravii e $P$. elegans. De fato, eqüinos intoxicados por essa duas plantas, desenvolvem, predominantemente, necrose coagulativa de túbulos uriníferos e, em menor extensão, degeneração hidrópico-vacuolar(Tokarnia et al. 1993, 1995).

Nos casos de intoxicação por Dichapetalum spp. na África, Gastrolobium spp., Oxylobium spp. e Acacia georginae na Austrália, outras plantas que contém o MF como principio ativo, não tem sido descritas especificamente essas alterações renais. Os comentários de Steyn (1928) sobre as lesões renais observadas em animais intoxicados por Dichapetalum cymosum mencionam áreas onde os túbulos tinham coloração mais fraca que os tecidos adjacentes e que essas lesões nos rins poderiam ser descritas como necrobiose localizada; esses comentários 
sugerem que a DHV também ocorre nesse tipo de intoxicação e que apenas não foi descrita como tal; o mesmo deve ter ocorrido no que se refere à intoxicação por Gastrolobium spp. e Oxylobium spp. uma vez que Gardner \& Bennetts (1956), mencionam, em relação a essas duas plantas, "alterações tóxicas, especialmente no epitélio dos túbulos contorcidos".

Por outro lado, nem todos os bovinos intoxicados por plantas que causam morte súbita desenvolvem a típica DHV. A nosso ver, esse fenômeno está relacionado à quantidade ingerida e ao tempo de evolução. Quanto maior a dose ingerida de $P$. marcgravii, mais curto é o período para aparecimento dos sintomas. Portanto, parece razoável que animais que ingiram maiores quantidades de MF, contido ou não em plantas, morram por parada cardíaca antes que a eliminação da substância tenha causado a lesão renal. Por outro lado, é possível que em algumas outras espécies animais, as lesões não ocorram ou não sejam tão nítidas.

A vacuolização de hepatócitos observada nesse estudo pode ou não estar relacionada ao efeito do MF, de vez que não se pode descartar que seja decorrente da anóxia determinada pela insuficiência cardíaca prévia à fase dramática da morte súbita. Essa lesão já foi relatada em intoxicação com plantas que causam morte súbita em bovinos, como Pseudocalymma elegans (Tokarnia et al. 1969, Helayel et al. 2009), Palicourea grandiflora (Döbereiner \& Tokarnia 1982), P. aeneofusca, P. juruana (Tokarnia \& Döbereiner 1982, Oliveira. et al. 2004), P. marcgravii (Tokarnia et al. 1990) e Arrabidaea bilabiata (Tokarnia et al. 2004). Lesão semelhante também foi observada em hepatócitos de coelhos e cobaios intoxicados experimentalmente por P. elegans (Tavares et al. 1974), e A. bilabiata (Döbereiner et al. 1984, Jabour et al. 2006), Mascagnia aff. rigida (Tokarnia et al. 1985) e P. marcgravii (Peixoto et al. 1987) em caprinos que receberam $P$. aeneofusca (Passos 1983) e P. marcgravii (Tokarnia et al. 1993), e em ovinos (Consorte et al. 1994) e equinos (Tokarnia et al. 1995) intoxicados por $P$. elegans.

Necrose hepática paracentral ou aleatória foi observada em parte dos bovinos intoxicados por MF e já foi relatada na intoxicação experimental por $A$. bilabiata em coeIhos (Jabour et al. 2006); há também referência à necrose na zona intermediária do lóbulo hepático na intoxicação experimental por $A$. bilabiata e $P$. elegans em coelhos (Döbereiner et al. 1984, Helayel et al. 2009). Essas lesões também podem ter sido determinadas pela estase / anóxia hepática ocasionada pela insuficiência cardíaca.

Infiltração inflamatória linfo-plasmocitária intersticial multifocal é uma lesão freqüentemente encontrada em rim de bovinos e não deve ser correlacionada à eliminação do MF, já que, sua instalação demanda mais tempo do que o período compreendido entre a ingestão do tóxico e a morte. Trata-se, mais provavelmente, de uma alteração conhecida como nefrite segmentar (Helayel et al. 2009).

Embora não seja muito comum, a morte aguda por deficiência de vitamina E/Selênio pode ser considerada no diagnóstico diferencial, apenas no que diz respeito à evolução clínica, já que os animais podem morrer sem apresentar sinais premonitórios ou após aparecimento súbito de depressão, dispnéia, taquicardia. A diferenciação deve ser feita durante a avaliação anátomo-histopatológica, uma vez que se observam lesões de caráter degenerativo-necrótico relacionadas à musculatura esquelética e, por vezes, ao miocárdio (Barros 2007), alterações não evidenciadas em animais intoxicados por MF. Em adição, a presença de DHV, ao exame microscópico dos rins, elimina quaisquer dúvidas.

A intoxicação por plantas cianogênicas também cursa com evolução superaguda e poderia ser confundida com intoxicação por MF, porém, o HCN é absorvido rapidamente e os sintomas da intoxicação aparecem logo após ou já durante a ingestão da planta (Clark \& Weiss 1952), diferentemente do que ocorre em bovinos intoxicados por MF. Além disso, não há sinais de insuficiência cardíaca e o exame microscópico não revela lesão significativa (Jones et al. 1983).

A intoxicação por Ricinus communis (folha e pericarpo), causa sintomas predominantemente neuromusculares $\mathrm{e}$ tem evolução superaguda de no mínimo algumas horas. Ao exame microscópico observa-se leve a acentuada vacuolização do parênquima hepático (Tokarnia et al. 2000).

A distinção entre o envenenamento por MF e plantas que causam morte súbita deve ser feita com base nos dados epidemiológicos, uma vez que não há quaisquer diferenças clínico-patológicas. A intoxicação criminosa por MF em bovinos é pouco provável, uma vez que a comercialização da substância foi proibida no Brasil. Não se pode perder de vista, contudo, que a toxidez do MF se mantém por décadas, desde que armazenado adequadamente (Eisler 1995) e também que, em 2004, dezenas de animais morreram no Zoológico de São Paulo envenenados pelo MF (Ortis 2005).

Com relação à ultraestrutura, confirmou-se que a lesão vista na microscopia óptica, de fato corresponde à vacuolização do citoplasma acompanhada por cariopicnose e que a formação dos vacúolos deve-se ao acúmulo de água. Por outro lado, o estudo não foi direcionado especificamente para análise ultraestrutural da lesão renal e a preservação do tecido foi limitada, o que dificultou a identificação de membranas ou organelas que pudessem dar origem ao vacúolo.

Por fim, este trabalho demonstra que a degeneração hidrópico-vacuolar dos túbulos uriníferos contorcidos distais e, eventualmente, túbulos coletores, associada à cariopicnose é uma lesão característica da intoxicação por MF em bovinos, o que indica que essa substância é o composto determinante ou, pelo menos, o mais importante na morte dos animais que ingerem plantas que causam morte súbita.

Agradecimentos.- Ao Sr. Geraldo Baêta, Embrapa Agrobiologia, pela ajuda com o material da microscopia eletrônica.

\section{REFERÊNCIAS}

Adesp 2007. Associação de Empresas Controladoras de Pragas do Estado. Disponível em <http://www.adesp.org.br> Acesso em 7.7.07.

Balcomb R., Bowen II C.A. \& Williamson H.O. 1983. Acute and sublethal effects of 1080 on starlings. Bull. Environ. Contam. Toxicol. 31:692-698. 
Barbosa J.D., Oliveira C.M.C., Tokarnia C.H. \& Riet-Corrêa F. 2003. Comparação da sensibilidade de bovinos e búfalos à intoxicação por Palicourea marcgravii (Rubiaceae). Pesq. Vet. Bras. 23(4):167-172.

Barnes R.A. \& Gilbert M.E.A. 1960. Investigação química preliminar de várias plantas brasileiras: Presença de alcalóides, saponinas e outras substâncias. Bolm Inst. Quím. Agríc. 58:9-26.

Barros C.S.L. 2007. Deficiência de selênio e vitamina E, p.257-263. In: Riet-Corrêa F., Schild A.L., Lemos R.A.A. \& Borges J.R. (Eds), Doença de Ruminantes e Equídeos. $3^{\underline{a}}$ ed. Editora Pallotti, Santa Maria.

Camargo W.A. 1962. Uma nova "erva-de-rato" tóxica para bovinos, Palicourea barbiflora: comparação com a Palicourea marcgravii var. pubescens e com Psychotria officinalis, Rubiaceae. Arqs Inst. Biológico, São Paulo, 29:1-11.

Cascon C.S. \& Mors W.B. 1962. Substâncias isoladas da Palicourea marcgravii St. Hil: uma nova síntese da N-metil-tiramina. Anais Assoc. Bras. Quím. 21:53-60.

Chenoweth M.B. \& Gilman A. 1946. Studies on the pharmacology of fluoroacetate. 1. Species response to fluoroacetate. J. Pharm. Exp. Therap. 87:90-103.

Chenoweth M.B. 1949. Monofluoroacetic acid and related compounds. J. Pharm. Exp. Therap. 97(4):383-424.

Chung H.M. 1984. Acute renal failure caused by acute monofluoroacetate poisoning. Vet. Hum. Toxicol. 26:29-32.

Clark R. \& Weiss K.E. 1952. Factors contributing towards bloat in ruminants. J. S. Afr. Vet. Med. Assoc. 23(2):103-106.

Clarke D.D. 1991. Fluoroacetate and fluorocitrate: Mechanism of action. Neurochem. Res. 16(9):1055-1058.

Coelho E.G., Amaral A.C.F., Ferreira J.L.P., Santos A.G., Pinheiro M.L.B. \& Silva J.R.A. 2007. Calcium oxalate crystals and methyl salicylate as toxic principles of the fresh leaves from Palicourea longiflora, an endemic species in the Amazon state. Toxicon 49:407-409.

Consorte L.B., Peixoto P.V. \& Tokarnia C.H. 1994. Intoxicação experimental por Pseudocalymma elegans (Bignoniaceae) em ovinos. Pesq. Vet. Bras. 14(4):123-133.

Costa M.V., Nascimento E.F., Pessoa J.M. \& Costa W.R. 1984. Lesões em bovinos intoxicados por Palicourea marcgravii St. Hil. Arq. Bras. Med. Vet. Zootec. 36(5):571-580.

Couceiro J.E.M., Silva A.C.C. \& Silva J.A. 1976. Observações e ensaios sobre a alegada intoxicação de bovinos por plantas, no Estado de Pernambuco. Congresso Brasileiro de Medicina Veterinária, Rio de Janeiro, p.45-46. (Resumo)

Cristofori P., Zanetti E., Fregona D., Piaia A. \& Trevisan A. 2007. Renal proximal tubule segment-specific nephrotoxicity: An overview on biomarkers and histopathology. Toxicol. Pathol. 35(2):270-275.

Cunha L.C., Gorniak S.L., Haraguchi M., Riet-correa F., Xavier F.G. \& Florio J.C. 2006 Palicourea marcgravii e Mascagnia rigida: um estudo por cromatografia em camada delgada (CCD). II Simpósio de Pós-Graduação e XV Semana Científica Prof. Dr. Benjamin Eurico Malucelli, USP, São Paulo. (Resumo em CD-ROM)

Döbereiner J. \& Tokarnia C.H. 1959. Intoxicação de bovinos pela "erva-de-rato" (Palicourea marcgravii St. Hil.) no vale do Itapicuru, Maranhão. Arqs Inst. Biol. Anim., Rio de J., 2:83-91.

Döbereiner J. \& Tokarnia C.H. 1982. Intoxicação experimental por Palicourea grandiflora (Rubiaceae) em coelhos. Pesq. Vet. Bras. 2(3):121-124.

Döbereiner J. \& Tokarnia C.H. 1983. Intoxicação experimental por Arrabidaea japurensis (Bignoniaceae) em coelhos. Pesq. Vet. Bras. 3(3):95-97.

Döbereiner J., Peixoto P.V. \& Tokarnia C.H. 1984. Intoxicação experimental por Arrabidaea bilabiata (Bignoniaceae) em coelhos. Pesq. Vet. Bras. 4(3):89-96.

Döbereiner J., Gava A., Consorte L.B. \& Tokarnia C.H. 1986. Intoxicação experimental por Mascagnia pubiflora (Malpighiaceae) em coeIhos. Pesq. Vet. Bras. 6(2):51-57.
Eisler R. 1995. Sodium monofluoroacetate (1080) hazards to fish, wildlife, and invertebrates: A synoptic review. Biological Report 27, Patuxent Environmental Science Centre, U.S. National Biological Service, Washington DC., USA, p.1-47.

Fillastre J.P. \& Raguenez-Viotte G. 1989. Cisplatin nephrotoxicity. Toxicol. Lett. 46(1/3):163-175.

Friedman M. \& Kaplan A. 1943. Studies concerning the site of renin formation in the kidney. IV. The renin content of the mammalian kidney following specific necrosis of proximal convoluted tubular epithelium. J. Exp. Med. 77:65-73.

Gagnin M.A.H. \& Maravalhas N. 1969. Ocorrência de alcalóides no gênero Palicourea. Anais do $20^{\circ}$ Congresso Nacional de Botânica, Goiânia, Goiás, p.91-105.

Gajdusek D.C. \& Luther G. 1950. Fluoroacetate poisoning: A review and report of a case. Am. J. Dis. Child. 79:310-320.

Gardner C.A. \& Bennetts H.W. 1956. The Toxic Plants of Western Australia. Western Australia Newspaper, Perth, p.83.

Gava A., Pilati C., Cristani J. Simões J. \& Simões L. 1998. Intoxicação cianogênica em bovinos alimentados com Tifton (Cynodon sp.). 8o CAMEV, Lages, SC, p.119. (Resumo)

González B., Suárez-Roca H., Bravo A., Salas-Auvert R. \& Avila D. 2000. Chemical composition and biological activity of extracts from Arrabidaea bilabiata. Pharm. Biol. 38(4):287-290.

Górniak S.L. 1988. Intoxicação por Palicourea marcgravii: uma abordagem experimental. Tese de Doutorado, Faculdade de Medicina Veterinária e Zootecnia, USP, São Paulo. 99p.

Gregg K., Hamdorf B., Henderson K., Kopecny J. \& Wong C. 1998. Genetically modified ruminal bacteria protect sheep from fluoroacetate poisoning. Appl. Environ. Microbiol. 64:3496-3498.

Guimarães C.C. 1934. Herva de rato. Vida Méd. 2:324-333.

Habermehl G. 1986. Comunicação pessoal. (Apud Górniak 1986)

Helayel M.A. 2008. Morte súbita em bovinos causada pela ingestão de Pseudocalymma elegans (Bignoniaceae) no município de Rio Bonito, RJ. Dissertação de Mestrado em Medicina Veterinária, Instituto de Veterinária, Universidade Federal Rural do Rio de Janeiro. 91p.

Helayel M.A., França T.N., Seixas J.N., Nogueira V.A., Caldas S.A. \& Peixoto P.V. 2009. Morte súbita em bovinos causada pela ingestão de Pseudocalymma elegans (Bignoniaceae) no município de Rio Bonito, RJ. Pesq. Vet. Bras. 29(7):498-508.

Jabour F.F., Seixas J.N., Tokarnia C.H. \& Brito M.F. 2006. Variação da toxidez de Arrabidaea bilabiata (Bignoniaceae) em resposta a coeIhos. Pesq. Vet. Bras. 26(3):171-176.

Jones T.C. \& Hunt R.D. 1983. Veterinary Pathology. $5^{\text {th }}$ ed. Lea and Febiger, Philadelphia, p.1443-1502.

Kemmerling W. 1996. Toxicity of Palicourea marcgravii: Combined effects of fluoroacetate, Nmethyltyramine and 2-methyltetrahydrobeta-carboline. Zeitschrift für Naturforschung 51(1/2):59-64.

Khattab F.K.I. 2007. Effects of sodium selenite on the ultrastructure of the kidney cortex in normal rats. J. Appl. Sci. Res. 3(9):803-810.

Krebs H.C., Kemmerling W. \& Habermehl G. 1994. Qualitative and quantitative determination of fluoroacetic acid in Arrabidaea bilabiata and Palicourea marcgravii by F-NMR spectroscopy. Toxicon 32:909-913.

Marais S.T. 1944. Monofluoroacetic acid, the toxic principle of "Gifblaar" Dichapetalum cymosum (Hook). Onderstepoort J. Vet. Sci. Anim. Ind. 20:67-73.

Maxie M.G. \& Robinson W.S.F. 2007. Cardiovascular system, p.1-105. In. Maxie M.G. (Ed.), Jubb, Kennedy, and Palmer's Pathology of Domestic Animals. Vol.3. $5^{\text {th }}$ ed. Saunders Elsevier, Philadelphia. 737p.

McEwan T. 1964. Isolation and identification of the principle of Gastrolobium grandiflorum. Queensl. J. Agric. Sci. 21(2):1-14.

McLIroy J.C. 1981. The sensivity of Australian animals to 1080 poison. I. Intraespecific variation and factors affecting acute toxicity. II. Marsupial and eutherian carnivores. Aust. Wildl. Res. 8:369-399. 
McLIroy J.C. 1982. The sensitivity of Australian animals to 1080 poison. IV. Native and introduced rodents. Aust. Wildl. Res. 9:505-517.

McLIroy J.C. 1984. The sensitivity of Australian animals to 1080 poison. VII. Native and introduced birds. Aust. Wildl. Res. 11:373-385.

Mello E.M.M. \& Fernandes J.S. 1940. Contribuição ao estudo de plantas tóxicas brasileiras. Serviço de Publicidade Agrícola, Ministério da Agricultura, Rio de Janeiro. 50p.

Morita H., Ichihara Y., Takeya K., Watanabe K., Itokawa H. \& Motidome M. 1989. A new alkaloid glycoside from the leaves of Palicourea marcgravii. Plant. Med. 55(3):288-289.

Oelrichs P.B. \& McEwan T. 1962. The toxic principle of Acacia georginae. Queensl. J. Agric. Sci. 19:1-16.

Oliveira M.M. 1963. Chromatographic isolation of monofluoroacetic acid from Palicourea marcgravii, St. Hil. Experientia 19:586.

Oliveira C.M.C., Barbosa J.D., Macedo R.S.C., Brito M.F., Peixoto P.V. \& Tokarnia C.H. 2004. Estudo comparativo da toxidez de Palicourea juruana (Rubiaceae) para búfalos e bovinos. Pesq. Vet. Bras. 24(1):27-30.

Ortis M. 2005. Comissão externa destinada a acompanhar as investigações sobre o envenenamento de animais ocorrido na Fundação Zoológico de São Paulo (Envenenamento no Zoológico de São Paulo). Disponível em <www.camara.gov.br/sileg/integras/292702.pdf> Acesso em 14.8.09.

Pacheco G. \& Carneiro V. 1932. Estudos experimentais sobre plantas tóxicas. I.Intoxicação dos animais pela "erva-de-rato da mata". Revta Soc. Paul. Med. Vet. 2(2/3):23-46.

Passos D.A. 1983. Intoxicação experimental em caprinos (Capra hircus) por Palicourea aeneofusca (M. Arg.) Standl (Rubiaceae). Dissertação de Mestrado em Ciência Veterinária, Universidade Federal Rural de Pernambuco, Recife. 40p.

Peckolt T. (Apud Guimarães 1934)

Peixoto, P.V. Döbereiner J., Tokarnia C.H. \& Peixoto C.S. 1987. Intoxicação experimental por Palicourea marcgravii (Rubiaceae) em coelhos. Pesq. Vet. Bras. 7(4):117-129.

Peters R.A. 1952. Lethal synthesis. Proc. R. Soc. Lond. B, Biol. Sci. 139(895):143-170.

Rodrigo R., Bosco C., Herrera P. \& Rivera G. 2004. Amelioration of myoglobinuric renal damage in rats by chronic exposure to flavonolrich red wine. Nephrol. Dial. Transplant. 19:2237-2244.

Schultz R.A., Coetzer J.A.W., Kellerman T.S. \& Naudé T.W. 1982. Observations on the clinical, cardiac and histopathological effects of fluoracetate in sheep. Onderstepoort. J. Vet. Res. 49:237-245.

Steyn D.G. 1928. Gifblaar poisoning. A summary of our present knowledge in respect of poisoning by Dichapetalum cymosum. Report Vet. Res. S. Afr. 13/14:187-194.

Tavares M.I., Rezende A.M.L. \& Döbereiner J. 1974. Intoxicação experimental por Pseudocalymma elegans em coelhos e cobaias. Pesq. Agropec. Bras. 9:91-94.
Tokarnia C.H., Döbereiner J., Canella C.F.C. \& Guimarães D.J. 1969. Intoxicação experimental por Pseudocalymma elegans (Vell.) Kuhlm. em bovinos. Pesq. Agropec. Bras. 4:195-204.

Tokarnia C.H., Döbereiner J. \& Silva M.F. 1981. Intoxicação por Palicourea grandiflora (Rubiaceae) em bovinos no Território de Rondônia. Pesq. Vet. Bras. 1(3):89-94.

Tokarnia C.H. \& Döbereiner J. 1982. Intoxicação experimental por Palicourea juruana (Rubiaceae) em bovinos e coelhos. Pesq. Vet. Bras. 2(1):17-20.

Tokarnia C.H., Döbereiner J. \& Couceiro J.M. 1983. Intoxicação por Palicourea aeneofusca (Rubiaceae), a causa de "mortes súbitas" em bovinos na Zona-da-Mata de Pernambuco. Pesq. Vet. Bras. 3(3):75-79.

Tokarnia C.H., Döbereiner J. \& Peixoto P.V. 1985. Intoxicação experimental por Mascagnia aff. rigida (Malpighiaceae) em bovinos no Norte do Espírito Santo. Pesq. Vet. Bras. 5(3):77-91.

Tokarnia C.H. \& Döbereiner J. 1986. Intoxicação por Palicourea marcgravii (Rubiaceae) em bovinos no Brasil. Pesq. Vet. Bras. 6(3):73-78.

Tokarnia C.H., Peixoto P.V. \& Döbereiner J. 1990. Poisonous plants affecting heart function of cattle in Brazil. Pesq. Vet. Bras. 10(1/2):110.

Tokarnia C.H., Peixoto P.V. \& Döbereiner J. 1991. Intoxicação experimental por Palicourea marcgravii (Rubiaceae) em caprinos. Pesq. Vet. Bras. 11(3/4):65-70.

Tokarnia C.H., Costa E.R., Barbosa J.D., Armién A.G. \& Peixoto P.V. 1993. Intoxicação experimental por Palicourea marcgravii (Rubiaceae) em eqüinos. Pesq. Vet. Bras. 13(3/4):67-72.

Tokarnia C.H., Peixoto P.V., Armién A.G., Barbosa J.D. \& Driemeier D. 1995. Intoxicação experimental por Pseudocalymma elegans (Bignoniaceae) em eqüinos. Pesq. Agropec. Bras. 15(1):35-39.

Tokarnia C.H., Döbereiner J. \& Peixoto P.V. 2000. Plantas que afetam o funcionamento do coração, p.19-46. In: Ibid. (Eds), Plantas Tóxicas do Brasil. Editora Helianthus, Rio de Janeiro.

Tokarnia C.H., Barbosa J.D., Oliveira C.M.C., Brito M.F., Oliveira R.B. \& Barbas L.A. 2004. Aspectos epidemiológicos e clínico-patológicos comparados da intoxicação por Arrabidaea bilabiata (Bignoniaceae) em búfalos e bovinos. Pesq. Vet. Bras. 24(2):74-79.

Tokarnia C.H. \& Döbereiner J. 1982. Comunicação pessoal (Universidade Federal Rural do Rio de Janeiro, Seropédica, RJ).

Tokarnia C.H. 2009. Comunicação pessoal (Universidade Federal Rural do Rio de Janeiro, Seropédica, RJ).

Twigg L.E. \& King D.R. 1991. The impact of fluoroacetate-bearing vegetation on native Australian fauna: A review. Oikos 61:412-430.

Vickery B. \& Vickery M.L. 1973. Toxicity for livestock of organofluorine compounds present in Dichapetalum plant species. Vet. Bull. 43(10):537-542. 